\title{
COMPARATIVE STUDY OF DIFFERENT METHODS FOR DOG SEMEN CRYOPRESERVATION AND TESTING UNDER CLINICAL CONDITIONS
}

\author{
F. SzÁSz ${ }^{1 *}$, G. GÁBOR ${ }^{2}$ and L. SOLTI ${ }^{1}$ \\ ${ }^{1}$ Department of Obstetrics and Reproduction, Faculty of Veterinary Science, Szent István \\ University, H-1400 Budapest, P.O. Box 2, Hungary; ${ }^{2}$ Department of Cattle Breeding, \\ Research Institute of Animal Breeding, Herceghalom, Hungary
}

(Received July 23, 1999; accepted May 3, 2000)

\begin{abstract}
The extenders and freezing rates from three different freezing protocols were combined and compared to each other in order to study the post-thawing acrosome integrity and fertility of frozen dog sperm. A commercial bovine TRIS-base extender (TRILADYL) and two self-made canine semen extenders (Norwegian and Dutch) were combined with a conventional bovine and two canine freezing regimes, and acrosome integrity of frozen/thawed spermatozoa was assessed by fluorescein isothiocyanate conjugated peanut agglutinin staining (FITC-PNA). Differences between freezing/thawing protocols were reflected in the proportion of cells with acrosomal damage and not based on motility results. It was concluded that during dog semen cryopreservation extenders had less influence on the post-thawing sperm quality than did the freezing rates. The optimal extender/freezing rate combination (TRILADYL/Norwegian) was used in the clinical practice to evaluate the fertility of frozen sperm administered by intrauterine insemination using a surgical approach. The pregnancy rate was $57 \%(4 / 7)$, but the average litter size was low (2.8). This may have been due to the insufficient sperm numbers contained in an insemination dose and/or to the incorrect timing of artificial insemination (AI). The final conclusion is that the commercial bovine extender is useful for freezing dog semen, and the TRILADYL/Norwegian freezing protocol is recommended as the most advantageous combination for the freezing of canine semen in the clinical practice.
\end{abstract}

Key words: Acrosome integrity, dog sperm, TRILADYL, FITC-PNA

Cryopreservation of canine semen is widely used for the long-term preservation of genetic material and for its transportation between countries. The type of semen extender and the specific freezing rate are important factors influencing the post-thawing sperm quality (sperm cell morphology and motility), which may influence the success rate of artificial insemination (AI). Since the first report of successful AI using frozen-thawed dog sperm was published (Seager, 1969), several cryopreservation methods have been developed and tested mostly

*Present address: H-2094 Nagykovácsi, Bánya u. 8, Hungary;

E-mail: fszasz@matavnet.hu; Fax: +36 (1) 425-3205 
under experimental conditions (Seager et al., 1975; Farstad, 1984; England, 1992; Linde-Forsberg, 1995; Silva and Verstegen, 1995). The most commonly used diluent for freezing canine semen is the TRIS-fructose-citric acid extender containing egg yolk and glycerol (Oettlé, 1986; Yubi et al., 1987; Farstad and Andersen-Berg, 1989; England, 1992; Dobrinski et al., 1993; Silva and Verstegen, 1995). Few studies have been conducted to evaluate commercial TRIS-base extenders, which have been routinely used for bovine semen preservation (Dobrinski et al., 1993; Silva and Verstegen, 1995). During the cooling and freezing/thawing process mammalian spermatozoa are subjected to several morphological and functional changes due to the damaging effect of ice crystallisation and osmolality imbalance (Mazur, 1985). The most frequently affected part of the spermatozoa is the acrosome (Oettlé, 1986), with the loss of acrosomal contents and swelling and rarefaction of the membrane (Rodriguez-Martinez et al., 1993). The sperm cell requires intact acrosome to perform acrosome reaction prior to penetrating the zona pellucida in order to fertilise the oocyte (Yanagimachi, 1981). Frozen/thawed dog sperm with damaged acrosome is unable to fertilise the oocyte, therefore the high rate of damaged cells decreases the fertilising capability of frozen semen.

The aims of the study were to investigate (1) the effect of different freezing protocols and extenders on the quality of frozen semen, (2) the acrosome integrity of spermatozoa examined by fluorescein staining (FITC-PNA), and (3) to test the frozen semen by intrauterine AI under clinical conditions.

\section{Materials and methods}

Experimental design

\section{Experiment I}

In the first part of the experiment (Exp. I) three freezing protocols and three different extenders were combined and compared to each other to examine postthawing motility and acrosome integrity by FITC-PNA staining in order to determine the optimal extender/freezing rate combination.

Semen preparation. Second-fraction ejaculates were collected (three times during 12 days) by manual stimulation from three Bull-Mastiff stud dogs (2-5 years of age). Samples with good sperm motility $(>90 \%)$ were pooled, divided into three equal parts and diluted with three different extenders: (A) TRILADYL ${ }^{\circledR}$ extender (MINITÜB, Tiefenbach, Germany), (B) Norwegian extender (TRIS $6.056 \mathrm{~g}$, citric acid $3.400 \mathrm{~g}$, fructose $2.500 \mathrm{~g}$, penicillin $2 \times 105 \mathrm{IU}$, dihydrostreptomycin $0.2 \mathrm{~g}$, glycerol $12 \mathrm{ml}$, egg yolk $2 \mathrm{ml}$, double-distilled water $184 \mathrm{ml}$ ), and (C) Dutch extender (TRIS $24.32 \mathrm{~g}$, glycocol $9.39 \mathrm{~g}$, citric acid $3.54 \mathrm{~g}$, glucose $7.50 \mathrm{~g}$, fructose $7.50 \mathrm{~g}, \mathrm{NaCl} 1.00 \mathrm{~g}, \mathrm{NaHCO}_{3} 1.5 \mathrm{~g}$, egg yolk $375 \mathrm{ml}$, glycerol $152.7 \mathrm{ml}$, penicillin $2 \times 106 \mathrm{IU}$, streptomycin $2 \mathrm{~g}$, ad $1500 \mathrm{ml}$ distilled water). Fi- 
nal sperm concentration was $150 \times 10^{6}$ cells $/ \mathrm{ml}$. Aliquots were equilibrated at $5{ }^{\circ} \mathrm{C}$ for $2 \mathrm{~h}$ in $10 \mathrm{ml}$ plastic tubes, then the samples were packed into $0.5 \mathrm{ml}$ mini straws (IMV Cassou, L'Aigle, France) under isothermic conditions and were frozen by programmable freezing equipment (DIGITCOOL-5300, IMV Cassou, L'Aigle, France). Before freezing, $500 \mu 1$ sperm was removed from each extended sample and fixed for evaluation of initial acrosome status by FITC-PNA staining. Three different protocols were used for freezing in each type of extender diluted samples: (1) Conventional bull semen freezing protocol: cooling from $+4{ }^{\circ} \mathrm{C}$ to $-10{ }^{\circ} \mathrm{C}$ at a freezing rate of $-4{ }^{\circ} \mathrm{C} / \mathrm{min}$, and further cooling from $-10{ }^{\circ} \mathrm{C}$ to $-140{ }^{\circ} \mathrm{C}$ at a rate of $-40^{\circ} \mathrm{C} / \mathrm{min}$. (2) Norwegian canine semen freezing protocol according to Farstad: cooling from $+5{ }^{\circ} \mathrm{C}$ to $-7^{\circ} \mathrm{C}$ at a rate of $-2{ }^{\circ} \mathrm{C} / \mathrm{min}$, then further cooling from $-7{ }^{\circ} \mathrm{C}$ to $-100{ }^{\circ} \mathrm{C}$ at a rate of $-50{ }^{\circ} \mathrm{C} / \mathrm{min}$, finally cooling from $-100{ }^{\circ} \mathrm{C}$ to $-180{ }^{\circ} \mathrm{C}$ at a freezing rate of $-25^{\circ} \mathrm{C} / \mathrm{min}$. (3) Dutch canine semen freezing protocol (Colenbrander, personal comm.): from $+5{ }^{\circ} \mathrm{C}$ to $-8{ }^{\circ} \mathrm{C}$ at a rate of $-5{ }^{\circ} \mathrm{C} / \mathrm{min}$, then from $-8{ }^{\circ} \mathrm{C}$ to $-80{ }^{\circ} \mathrm{C}$ at a freezing rate of $-35{ }^{\circ} \mathrm{C} / \mathrm{min}$ and finally from $-80{ }^{\circ} \mathrm{C}$ to $-140{ }^{\circ} \mathrm{C}$ at a freezing rate of $-30^{\circ} \mathrm{C} / \mathrm{min}$. At the end of each freezing protocol the samples were plunged into liquid nitrogen $\left(\mathrm{LN}_{2}\right)$.

Estimation of motility after the freezing/thawing procedure. Ten straws of each extender/freezing rate combinations were thawed in waterbath according to the appropriate recommendations $\left(1:\right.$ at $38^{\circ} \mathrm{C}$ for $50 \mathrm{sec}$; 2 : at $70{ }^{\circ} \mathrm{C}$ for $8 \mathrm{sec}$; 3: at $38^{\circ} \mathrm{C}$ for $50 \mathrm{sec}$ ), and motility was evaluated visually by phase-contrast microscope (NIKON OPTIPHOT, Japan) equipped with a heating plate. Five fields were evaluated in a blind manner.

Assessment of acrosome integrity by FITC-PNA staining. After motility examination, aliquots from each extender/freezing rate combinations were diluted with modified Tyrode's medium (Parrish et al., 1988) at a rate of 1:10 and centrifuged with $300 \mathrm{~g}$ for $5 \mathrm{~min}$. Each sperm pellet was resuspended in $1 \mathrm{ml}$ modified Tyrode's medium and assessed for acrosome integrity as described below. Sperm ( $200 \mu 1 /$ straws) were subsequently fixed for $10 \mathrm{~min}$ by addition of $200 \mu 1$ modified Tyrode's medium containing 4\% (w/v) paraformaldehyde and 1\% (w/v) glutaraldehyde. After washing (centrifugation with $300 \mathrm{~g}$ for $5 \mathrm{~min}$ ) the pellet was resuspended in $100 \mu 1$ modified Tyrode's medium and stained with $100 \mu 1$ of $100 \mu \mathrm{g} / \mathrm{ml}$ FITC-PNA (EY Laboratories, Inc., San Mateo, LA, USA) for 30 min at $38{ }^{\circ} \mathrm{C}$. A drop of $20 \mu \mathrm{l}$ stained sperm suspension was smeared on a coverslip and air-dried in the dark. Specimens were examined under an epifluorescence microscope (BH2RFCA, Olympus, Tokyo, Japan) equipped with DMU set of filters, and 200 cells were evaluated by FITC-PNA staining (Szász et al., 1997). Sperm cells with an intact acrosome (AI) were not stained with FITC-PNA while cells with acrosome damage (AD) were either intensively stained with FITC-PNA or showed patchy fluorescence in this region. Cells that had completed the acrosome reaction (AR) showed exclusive equatorial labelling of FITC-PNA with different fluorescent intensity due to loss of PNA binding sites. All slides were scored in a blind manner. 
SZÁSZ et al.

\section{Experiment II}

Use of frozen dog semen under clinical conditions. In this part of the study the extender/freezing rate combination found to be the most advantageous in Exp. I (TRILADYL/Norwegian freezing rate, 2/A) was tested under clinical conditions using clinical patients for the inseminations.

Animals. Seven bitches of different breeds (mongrel, French bulldog, Beagle, Doberman) of 2 to 5 years of age were included in the experiment. Semen used for insemination was collected from valuable stud dogs of the appropriate breed, and was frozen and evaluated as described above.

Timing of the insemination. Vaginal smears were collected and stained by Shorr-Papanicolaou staining (Niemand and Suter, 1994) from the 3rd-4th day of pro-oestrus until the onset of cytological oestrus, then samples were taken every day until the time of insemination. Additionally, blood samples ( $3 \mathrm{ml}$ into heparinized tubes) were collected from the v. cephalica antebrachii to measure the plasma progesterone (P4) concentration by microplate enzyme-immunoassay (ELISA). The inseminations were performed according to the plasma P4 level $(8.5-12 \mathrm{ng} / \mathrm{ml})$.

Intrauterine insemination using surgical technique. The surgical procedure (laparotomy) was performed in dorsal recumbency under general anaesthesia with a fentanyl $0.5 \mathrm{mg} / \mathrm{kg}$ b.w. (Fentanyl inj., G. Richter Co. Ltd., Budapest, Hungary) + midazolam $2 \mathrm{mg} / \mathrm{kg}$ b.w. (Dormicum inj., EGIS Co. Ltd., Budapest, Hungary) combination. The hair from the ventral abdomen was clipped, and after routine surgical preparation a $4-5 \mathrm{~cm}$ incision was made midway between the umbilicus and the pubis through the linea alba. The uterus was elevated and a venous catheter (Vasocan, Branüle 22G 1", B. BRAUN-Melsungen AG, Germany) was inserted into the lumen of the uterine body. A syringe containing $2 \mathrm{ml}$ of semen was attached to the catheter and the semen was slowly injected into the uterus. After removing the catheter, sterile moistened gauze was held over the site of injection, then the uterus was replaced into the abdomen and the wound was closed using a routine method.

Pregnancy diagnosis. The animals were checked for pregnancy by ultrasonography with a $5 \mathrm{MHz}$ probe (Pie-Medical Vet Scanner 250, PIE MEDICAL EQUIPMENT B.V., Maastricht, The Netherlands) between days 24 and 30 after insemination.

\section{Statistical analyses}

All statistical analyses were conducted with the Statistical Analysis System (Cary, NC). Two-sample Student's $t$-tests were used to determine the effect of freezing protocols and extenders on acrosome integrity and semen motility (SAS, 1990). 


\section{Results}

In Experiment I significant differences $(\mathrm{P}<0.001)$ were found in acrosome integrity between the different extender/freezing rate combinations (Table 1 ). The most serious damaging effect (acrosome intact sperm $<50 \%$ ) was observed in samples that were frozen using the bull sperm freezing protocol $(\mathrm{A} / 1$, $\mathrm{B} / 1, \mathrm{C} / 1$ ). The highest incidence of acrosome lost sperm (AR) was also found in the samples that were frozen according to bull protocol $(14 \pm 3 \%, 17 \pm 2 \%$, $33 \pm 4 \%$ ). Focusing only on the effect of extender on sperm acrosome integrity after freezing, no significant differences were detected between the extenders used (Table 1). Post-thawing sperm motility was reduced in average $35 \%$ of initial motility, except in case of the $\mathrm{A} / 1$ and $\mathrm{C} / 1$ combinations where the motility reduction was higher (the ratio of motile sperm remained under $40 \%$ ). Acrosome status did not correlate well with motility rate.

\section{Table 1}

Post-thawing motility and acrosome status of sperm after using different combinations of extenders and freezing rates

\begin{tabular}{|c|c|c|c|c|c|c|c|c|c|}
\hline \multirow{2}{*}{ Parameters tested } & \multicolumn{9}{|c|}{ Extender/freezing rate combinations } \\
\hline & $\mathrm{A} / 1$ & $\mathrm{~A} / 2$ & $\mathrm{~A} / 3$ & $\mathrm{~B} / 1$ & $\mathrm{~B} / 2$ & $\mathrm{~B} / 3$ & $\mathrm{C} / 1$ & $\mathrm{C} / 2$ & $\mathrm{C} / 3$ \\
\hline $\begin{array}{l}\text { Acrosome intact sperm rate } \\
\text { before freezing }\end{array}$ & 91 & 90 & 92 & 90 & 90 & 92 & 91 & 92 & 91 \\
\hline Post-thawing parameters & & & & & & & & & \\
\hline Acrosome intact sperm rate & $49 \pm 2$ & $66 \pm 2$ & $61 \pm 2$ & $47 \pm 3$ & $65 \pm 2$ & $61 \pm 4$ & $47 \pm 2$ & $63 \pm 2$ & $68 \pm 2$ \\
\hline Acrosome damaged sperm rate & $37 \pm 1$ & $26 \pm 2$ & $32 \pm 2$ & $36 \pm 2$ & $31 \pm 3$ & $37 \pm 3$ & $20 \pm 2$ & $26 \pm 3$ & $27 \pm 2$ \\
\hline Acrosome reacted sperm rate & $14 \pm 3$ & $8 \pm 2$ & $7 \pm 2$ & $17 \pm 2$ & $4 \pm 2$ & $2 \pm 1$ & $33 \pm 4$ & $11 \pm 2$ & $5 \pm 2$ \\
\hline Post-thawing motility (\%) & 35 & 50 & 40 & 40 & 50 & 50 & 30 & 50 & 40 \\
\hline
\end{tabular}

Extender/freezing rate combinations:

A/1: TRILADYL/Bull freezing protocol; A/2: TRILADYL/Norwegian protocol; A/3: TRILADYL/ Dutch protocol

B/1: Norwegian extender/Bull freezing protocol; B/2: Norwegian extender/Norwegian protocol; B/3: Norwegian extender/ Dutch protocol

C/1: Dutch extender/Bull freezing protocol; $\mathrm{C} / 2$ : Dutch extender/Norwegian protocol; $\mathrm{C} / 3$ : Dutch extender/Dutch protocol

According to the results of Exp. I the A/2 (TRILADYL/Norwegian protocol) extender/freezing rate combination was used for freezing semen from stud dogs and for testing it in the clinical practice (Exp. II). Surgical artificial inseminations were performed only once per bitch on the day of detecting the optimal blood progesterone level (Fig. 1). The pregnancy rate was $57 \%(4 / 7)$, but the average litter size was relatively low (2.8) (Table 2). 


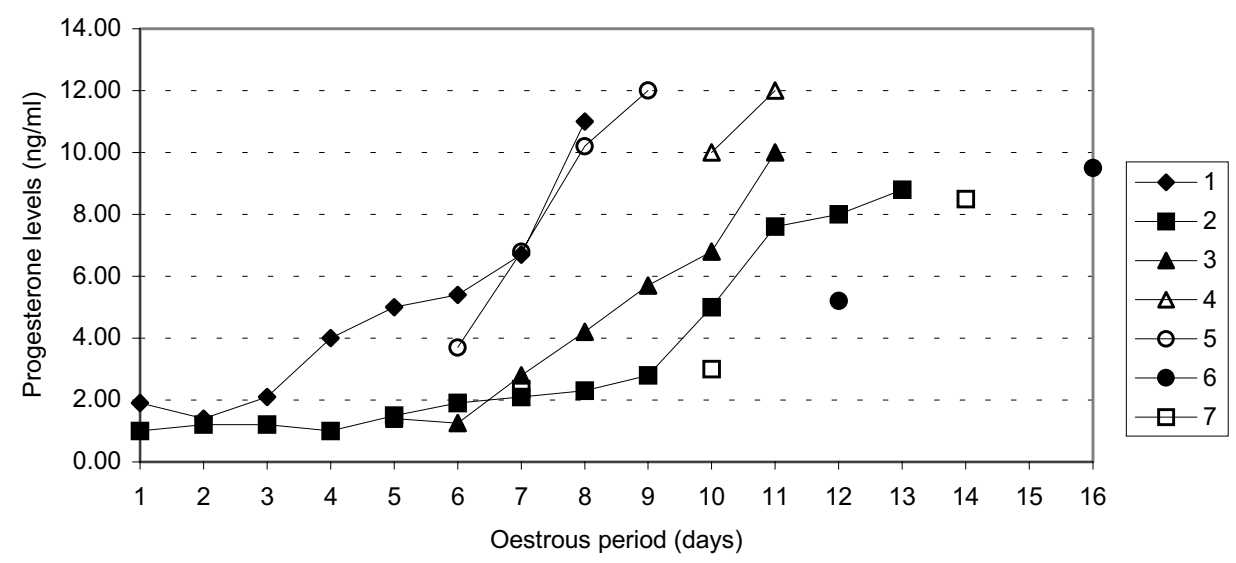

Fig. 1. Serum progesterone concentrations of inseminated females no. 1-7 during the oestrous period. The last $\mathrm{P} 4$ values indicate the day of surgical AI as well

Table 2

Serum progesterone concentrations on the day of AI and pregnancy diagnosis of inseminated females

\begin{tabular}{ccccc}
\hline $\begin{array}{c}\text { Inseminated } \\
\text { bitches }\end{array}$ & $\begin{array}{c}\text { Day of AI from } \\
\text { onset of oestrus }\end{array}$ & $\begin{array}{c}\text { Serum P4 level } \\
\mathrm{ng} / \mathrm{ml}\end{array}$ & $\begin{array}{c}\text { Pregnancy } \\
\text { diagnosis }\end{array}$ & Litter size \\
\hline 1 & 8 & 11 & pregnant & 2 \\
2 & 13 & 8.8 & pregnant & 4 \\
3 & 11 & 10 & pregnant & 2 \\
4 & 11 & 12 & non-pregnant & \\
5 & 9 & 12 & non-pregnant & 3 \\
7 & 16 & 9.5 & non-pregnant & pregnant \\
\hline
\end{tabular}

\section{Discussion}

With the improvement of AI and semen freezing technology the breeders' interest in $\mathrm{AI}$ and cryopreservation has grown exponentially and canine semen banks have been established. However, this commercialisation has led to a situation in which much of the information about extenders and freezing protocols remains proprietary. The objectives of this study were to examine semen extender/freezing rate combinations based on post-thawing acrosome morphology, and finally the most advantageous combination was tested under clinical conditions. A further aim was to find a standard semen preservation protocol for the routine clinical work. 
As the extender may have an impact on sperm morphology and viability during the freezing/thawing procedure, several authors have studied and compared different compositions. Battista et al. (1988) evaluated TRIS-, TES-, lactose- and PIPES-based extenders by examining post-thawing motility and longevity, and observed the most favourable values with the TRIS-based extender. Similar results were found by Olar et al. (1989) and England (1992) who experienced the best survival rate of spermatozoa after freezing in TRIS- or TES/TRISbased extenders. However, as the experimentally developed and self-made extenders are difficult to standardise and the repeatability of results is poor, the clinicians' interest turned to commercial semen extenders used for other species. Two bovine semen extenders (TRILADYL and IMV Universal) were compared to TRIS-based and PIPES-based extenders by Dobrinski et al. (1993), using three different freezing protocols and estimating the post-thawing motility of canine semen. The most vigorous motility was found in TRILADYL and IMV Universal diluted samples. Silva and Verstegen (1995) also used bovine semen extenders (Laiciphos and Biociphos) for freezing dog semen, and in vivo fertilising ability was assessed by inseminating bitches under experimental conditions. High post-thawing motility and pregnancy rate $(60 \%$ and $100 \%$, respectively) were reported. In the first part of the present experiment it was realised that the TRILADYL extender was appropriate for the cryopreservation of canine semen. It was concluded that extenders had less influence on the post-thawing sperm quality than did the freezing rates. These differences were reflected in the ratio of cells with acrosomal damage and were not based on motility results. The effect of freezing regime on the acrosomal membrane was clearly demonstrated, namely the intact acrosome rate was more than $15 \%$ lower after using the twostep freezing protocol (bull semen freezing protocol). Similar conclusions were drawn by Dobrinski et al. (1993), who found a significant effect of freezing rate on the post-thawing semen quality, but these observations were based only on the semen motility parameters after freezing. Several methods have been used for evaluating the morphological or morphofunctional state of spermatozoa after freezing. Oettlé (1986) reported approx. 45\% sperm with normal acrosome using differential staining for light microscopy evaluation. Similar results $(45-55 \%$ acrosome intact sperm rates) were reported by Ivanova-Kicheva et al. (1995) who examined the acrosome integrity by Congo red-eosin-gentian violet staining after two different thawing regimes. The higher rate of acrosome intact cells may be due to the more considerate freezing/thawing protocol and/or the more accurate examination method using FITC-PNA fluorescence staining technique.

The only way to receive direct information about the fertility of frozen/thawed canine semen is to use it for insemination. Several publications appeared on the fertility of frozen dog sperm based on pregnancy results (Platz and Seager, 1977; Fontbonne and Badinand, 1993; Silva and Verstegen, 1995). Most of them examined the semen under experimental conditions, presenting high 
pregnancy rates $(92-100 \%)$. Few reports are available on conception rates achieved with frozen canine semen in the clinical practice; however, the variability of inseminated bitches affects the success of AI, which gives the most reliable information about the usability of this method. Seager et al. (1975) reported fluctuating pregnancy rates $(9.5 \%-63.6 \%)$ over a five-year period using pelleted frozen semen in different breeds. A $67 \%$ pregnancy result was presented by Farstad (1984) after insemination of 92 bitches with frozen semen using nonsurgical intrauterine insemination under clinical conditions. During four years, $45.7 \%$ of bitches whelped after frozen-semen AI registered by the Swedish Kennel Club performing non-surgical intrauterine inseminations (Linde-Forsberg, 1995). A pregnancy rate of approximately $60 \%$ was reported by Olar (1984) using surgical intrauterine insemination technique; however, the number of animals was limited. Comparing the $57 \%$ pregnancy result achieved in this study to pregnancy rates published in the literature, it can be concluded that the examined extender/freezing protocol combination is acceptable for the clinical practice. Moreover, the advantage of using TRILADYL extender is the easy preparation and standardisation of the dilution process, which decreases the possibility of artificial effects.

The relatively low litter size (2.8) might be due to the incorrect timing of AI, the insemination of virgin dogs or the inappropriate site of insemination. Namely, Tsutsui et al. (1989) found that more than $20 \times 10^{6} / 0.1 \mathrm{ml}$ sperm had to be inseminated into the ipsilateral uterine horn to fertilise ova ovulated on the contralateral side also. Improving the accuracy of the timing of ovulation, examining the number of ovulated follicles on the ovaries, or inseminating both uterine horns may increase the number of puppies born.

In summary: we found significant differences between freezing rates when examining the post-thawing acrosome integrity of spermatozoa, but did not see a significant effect of extenders on sperm morphology after freezing. The TRILADYL/Norwegian freezing protocol is recommended as the combination most advantageous for freezing canine semen in the clinical practice.

\section{Acknowledgements}

The authors thank Mrs Anna Wölfling for hormonal assays, the National AI Centre for the use of equipment, and WALTHAM for providing dog foods and financial su pport.

\section{References}

Battista, M., Parks, J. and Concannon, P. (1988): Canine sperm post-thaw survival following freezing in straws or pellets using PIPES, Lactose, TRIS or TEST extenders. XIth International Congress on Animal Reproduction and Artificial Insemination (Dublin) 3, 229-231. 
Dobrinski, I., Lulai, C., Barth, A. D. and Post, K. (1993): Effects of four different extenders and three different freezing rates on post-thaw viability of dog semen. J. Reprod. Fert. Suppl. 47, 291-296.

England, G. C. W. (1992): The Cryopreservation of Dog Semen. PhD Thesis, The Royal Veterinary College, University of London.

Farstad, W. (1984): Bitch fertility after natural mating and artificial insemination with fresh or frozen semen. J. Small Anim. Pract. 25, 561-565.

Farstad, W. and Andersen-Berg, K. (1989): Factors influencing the success rate of artificial insemination with frozen semen in the dog. J. Reprod. Fert. Suppl. 39, 289-292.

Fontbonne, A. and Badinand, F. (1993): Canine artificial insemination with frozen semen: Comparison of intravaginal and intrauterine deposition of semen. J. Reprod. Fertil. Suppl. 47, 325-327.

Ivanova-Kicheva, M. G., Subev, M. S., Bobadov, N. D., Dacheva, D. P. and Rouseva, R. (1995): Effect of thawing regimens on the morphofunctional state of canine spermatozoa. Theriogenology 44, 563-569.

Linde-Forsberg, C. (1995): Artificial insemination with fresh, chilled extended, and frozen-thawed semen in the dog. Sem. Vet. Med. Surg. 10, 48-58.

Mazur, P. (1985): Basic concepts of freezing cells. Proceedings of the First International Conference on Deep Freezing of Boar Semen, Uppsala, pp. 91-111.

Niemand, H. G. and Suter, P. F. (1994): Praktikum der Hundeklinik. Blackwell, Berlin.

Oettlé, E. E. (1986): Changes in acrosome morphology during cooling and freezing of dog semen. Anim. Reprod. Sci. 12, 145-150.

Olar, T. T. (1984): Cryopreservation of dog spermatozoa. PhD Thesis, Colorado State University.

Olar, T. T., Bowen, R. A. and Pickett, B. W. (1989): Influence of extender, cryopreservative and seminal processing procedures on post thaw motility of canine spermatozoa frozen in straws. Theriogenology 31, 451-461.

Parrish, J. J., Susko-Parrish, J., Winer, M. A. and First, N. L. (1988): Capacitation of bovine sperm by heparin. Biol. Reprod. 38, 1171-1180.

Platz, C. C. and Seager, S. W. J. (1977): Successful pregnancies with concentrated frozen canine semen. Lab. Anim. Sci. 27, 1013-1016.

Rodriguez-Martinez, H., Ekwall, H. and Linde-Forsberg, C. (1993): Fine structure and elemental composition of fresh and frozen dog spermatozoa. J. Reprod. Fert. Suppl. 47, 279-285.

Seager, S. W. J. (1969): Successful pregnancies utilizing frozen dog semen. AI Digest 17, 6-7.

Seager, S.W. J., Platz, C. C. and Fletcher, W. S. (1975): Conception rates and related data using frozen dog semen. J. Reprod. Fert. 45, 189-192.

Silva, L. D. M. and Verstegen, J. P. (1995): Comparisons between three different extenders for canine intrauterine insemination with frozen-thawed spermatozoa. Theriogenology 44, 571-579.

Szász, F., Cheng, F. P., Marks, A., Colenbrander, B. and Solti, L. (1997): Induction of acrosome reaction in dog sperm by calcium ionophore. Acta Vet. Hung. 45, 177-187.

Tsutsui, T., Shimizu, T., Ohara, N., Shiba, Y., Hironaka, T., Orima, H. and Ogasa, A. (1989): Relationship between the number of sperms and the rate of implantation in bitches inseminated into unilateral uterine horn. Jpn. J. Vet. Sci. 51, 257-263.

Yanagimachi, R. (1981): Mechanisms of fertilisation in mammals. In: Mastroianni, L., Biggers, J. D. (eds) Fertilization and Embryonic Development In Vitro. Plenum Press, New York, pp. 81-182.

Yubi, C. A., Ferguson, J. M., Renton, J. P., Harker, S., Harvey, M. J. A., Bagyjenji, B. and Douglas, T. A. (1987): Some observations on the dilution, cooling and freezing of canine semen. J. Small Anim. Pract. 28, 753-761. 